Annuaire suisse de politique de développement

23-1 | 2004

Faits et statistiques 2004

\title{
12. Politique d'asile et questions migratoires
}

\section{Rosita Fibbi}

\section{OpenEdition}

\section{Journals}

Édition électronique

URL : http://journals.openedition.org/aspd/482

DOI : 10.4000/aspd.482

ISSN : 1663-9669

\section{Éditeur}

Institut de hautes études internationales et du développement

\section{Édition imprimée}

Date de publication : 1 avril 2004

Pagination : 209-224

ISSN : 1660-5934

\section{Référence électronique}

Rosita Fibbi, «12. Politique d'asile et questions migratoires », Annuaire suisse de politique de

développement [En ligne], 23-1 | 2004, mis en ligne le 16 mars 2010, consulté le 08 septembre 2020 URL : http://journals.openedition.org/aspd/482 ; DOI : https://doi.org/10.4000/aspd.482 


\section{Politique d'asile et questions migratoires*}

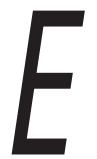

N DÉPIT de ses dimensions somme toute limitées sur le plan quantitatif, la question de l'asile est à l'origine d'une intense activité sur le plan politique, avec la révision de la loi, les répercussions dans ce domaine du plan d'allègement budgétaire, l'accélération de la procédure et l'élargissement du principe de l'Etat tiers. La loi sur les étrangers et la loi sur la naturalisation, mises en route dans les années précédentes, n'ont pas encore achevé leur parcours institutionnel. On enregistre cette année l'entrée du pouvoir judiciaire dans le large débat public avec un jugement retentissant affirmant le principe de non-discrimination envers les étrangers en matière de naturalisation. Le changement de climat politique consécutif aux élections dessine de nouveaux rapports de force au Parlement et au Conseil fédéral qui laissent augurer une radicalisation de la politique d'asile en particulier et de la politique de migration en général.

\subsection{Politique d'asile}

Demandes d'asile déposées

En 2002, quelque 26'000 personnes ont déposé une demande d'asile en Suisse: ceci représente une augmentation de $26 \%$ par rapport à l'année précédente, qui se situe légèrement au-dessus de la moyenne des dix dernières années (graphique 1).

\section{Graphique 1: Evolution des demandes d'asile, 1991-2002}

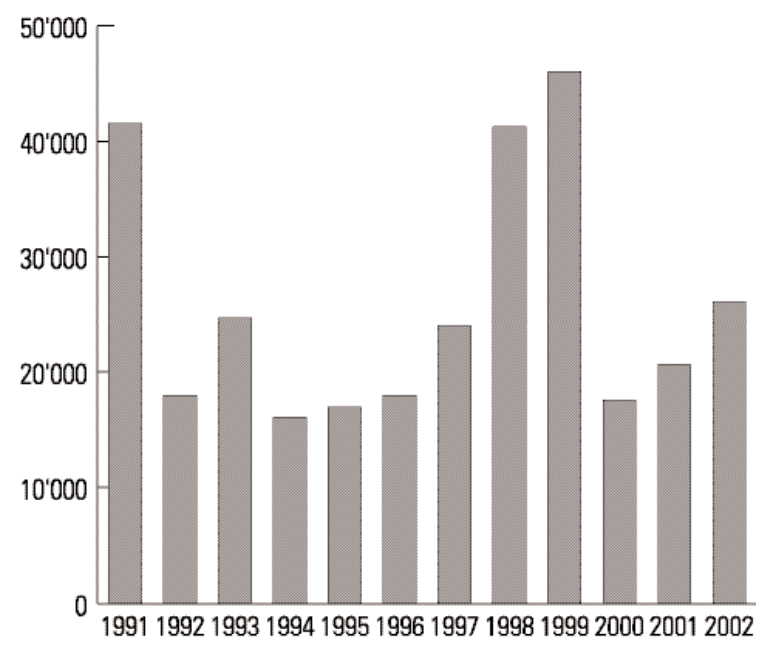

Source: ODR, Statistique en matière d'asile 2002, janvier 2003.

* Par Rosita Fibbi, Institut d'anthropologie et sociologie, Université de Lausanne; Forum suisse pour l'étude des migrations et de la population, Université de Neuchâtel. 
Comme par le passé, les groupes de demandeurs d'asile les plus importants numériquement proviennent de la région balkanique: $14 \%$ de la RF de Yougoslavie, $6 \%$ de la Bosnie-Herzégovine et 4,2\% de la Macédoine; ils sont suivis par la Turquie $(7,4 \%)$ et l'Irak $(4,5 \%)$.

Tableau 36: Evolution des demandes d'asile 2001-2002 selon le pays d'origine

\begin{tabular}{llrrr}
\hline Rang & Pays & Demandes d'asile 2001 & Demandes d'asile 2002 & Total en \% \\
\hline 1 & RF Yougoslavie & 3425 & 3692 & 14.1 \\
\hline 2 & Turquie & 1960 & 1940 & 7.4 \\
\hline 3 & Bosnie et Herzégovine & 1230 & 1548 & 5.9 \\
\hline 4 & Irak & 1201 & 1182 & 4.5 \\
\hline 5 & Macédoine & 884 & 1085 & 4.2 \\
\hline 6 & Nigéria & 289 & 1062 & 4.1 \\
\hline 7 & Algérie & 828 & 1020 & 3.9 \\
\hline 8 & Roumanie & 33 & 968 & 3.7 \\
\hline 9 & Angola & 600 & 824 & 3.2 \\
\hline 10 & Bulgarie & 229 & 785 & 3.0 \\
\hline Autres nations & 9954 & 12019 & 46.0 \\
\hline Total & $\mathbf{2 0 6 3 3}$ & $\mathbf{2 6 1 2 5}$ & $\mathbf{1 0 0 . 0}$ \\
\hline
\end{tabular}

Source: ODR, Statistique en matière d'asile 2002, janvier 2003.

Plusieurs pays figurent pour la première fois parmi les dix pays de départ les plus importants pour les requérants d'asile: le Nigeria (4,1\%), l'Angola (3,2\%) ainsi que la Roumanie $(3,7 \%)$ et la Bulgarie (3\%).

Contrairement à ce qui a été avancé dans le vif du débat public autour de l'initiative UDC en novembre 2002, la Suisse ne figure pas en tête des pays européens par nombre de demandes d'asile rapporté à la population, mais elle arrive en troisième position, derrière l'A Autriche et la Norvège (graphique 2).

Graphique 2: Demandes d'asile par rapport à 100'000 habitants en Europe de l'Ouest, $1^{\text {er }}$ décembre 2001-30 juin 2002

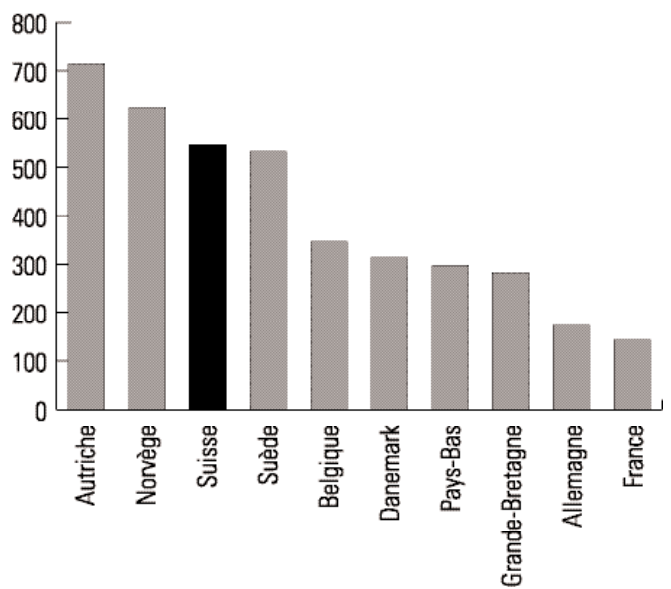

Source: ODR, Statistique en matière d'asile 2002, janvier 2003. 


\section{$\square$ Octroi de l'asile}

En 2002, l'Office fédéral des réfugiés (ODR) a octroyé l'asile à 1729 personnes, soit 23,3\% de moins que l'année précédente. Ainsi, le taux moyen de reconnaissance de la qualité de réfugié sur le total des décisions intervenues est passé de $11,7 \%$ à $8 \%$. Ce taux moyen recouvre des taux très variables d'une nationalité à 1'autre: supérieurs à $30 \%$ pour les ressortissants iraquiens et turcs, supérieur à $15 \%$ pour les Sri lankais et proches de $5 \%$ pour les Bosniaques et les Congolais. $70 \%$ de ces décisions sont intervenues au titre du regroupement familial.

Quelque 4170 personnes ont bénéficié d'une admission provisoire en 2002 (53\% de moins qu'en 2001), prolongeant ainsi la tendance à la baisse qui s'est amorcée depuis 1999; ce sont essentiellement des ressortissants de la RF de Yougoslavie et de Bosnie-Herzégovine. Les cas réglés par la police des étrangers (8725) sont également en diminution par rapport à l'an dernier $(-20 \%)^{1}$. Globalement, les personnes relevant du domaine de l'asile sont restées stables $(+0,4)$ d'une année sur l'autre.

Tableau 37: Personnes relevant du domaine de l'asile (état à fin décembre 2001 et 2002)

\begin{tabular}{lrrr}
\hline & $\mathbf{3 1 . 1 2 . 2 0 0 1}$ & $\mathbf{3 1 . 1 2 . 2 0 0 2}$ & Variation (\%) \\
\hline Effectif total & 93363 & 93741 & $\mathbf{0 . 4}$ \\
\hline Réfugiés reconnus & 26577 & 26034 & -2.0 \\
\hline Admissions provisoires & 30734 & 26332 & -14.3 \\
\hline Traitement/exécution en suspens ou bloqué & 10398 & 13420 & 29.1 \\
\hline Cas en suspens en première instance (ODR) & 14603 & 16184 & 10.8 \\
\hline Cas en suspens en deuxième instance (CRA) & 11051 & 11771 & 6.5 \\
\hline
\end{tabular}

Source: ODR, Statistique en matière d'asile 2002, janvier 2003.

En 2002, on a enregistré 17'000 départs de Suisse, un chiffre en légère augmentation par rapport à l'année précédente $(+7,4 \%)$. Les départs non officiels constituent, comme à l'accoutumée, plus de la moitié des départs. Sont classés comme départs non officiels le retour volontaire mais non annoncé, la réémigration vers un pays tiers ainsi que la permanence en Suisse sans documents valables (sans-papiers).

L'asile a été révoqué pour 3188 réfugiés reconnus, plus aucun danger ne menaçant ces personnes. Par ailleurs, l'ODR a levé l'admission provisoire pour 9219 personnes, la plupart des ressortissants du Sri Lanka et de l'ancienne Yougoslavie ayant bénéficié d'une réglementation cantonale.

\section{$\square$ Décisions fédérales en matière d'asile}

La dernière revue de la politique d'asile se terminait avec la nouvelle du rejet timide de l'initiative UDC dite «contre les abus dans le droit d'asile» (Annuaire $2003, n^{\circ} 1$, p. 216), qui laissait prévoir un tour de vis en matière d'asile, notamment lors de la discussion aux Chambres fédérales des diverses lois ayant trait à l'asile et aux étrangers. Les retombés de la votation ont été plutôt indirectes. Nous en citerons deux, à la portée fort différente: la nomination à la tête de la Commission fédérale des réfugiés et surtout le plan d'assainissement budgétaire de la Confédération.

1 Rappelons que depuis l'année 2000 ces personnes ne figurent plus dans la statistique des personnes relevant de l'asile. 
Estimant que la Commission fédérale des réfugiés devait être indépendante de l'Office fédéral des réfugiés, le directeur de l'ODR, Jean-Daniel Gerber, souhaitait quitter depuis longtemps le poste de président de cette commission. Ainsi, au lendemain des votations de l'automne 2002, les autorités ont décidé de donner suite à ce souhait et ont choisi un nouveau président en la personne de Roland Eberle, un politicien UDC à l'origine de l'initiative, repoussée le 24 novembre, qui cherchait à renvoyer les requérants d'asile ayant transité par un Etat sûr. La nomination de ce conseiller d'Etat thurgovien, avec effet au $1^{\text {er }}$ janvier 2003, indique la volonté des autorités d'impliquer davantage l'UDC dans la prise de décision en matière d'asile. La commission, qui jusqu'ici s'était tenue à son rôle avant tout consultatif, acquiert ainsi un nouveau profil politique.

\section{Le paquet d'allègement budgétaire}

A la fin de l'année 2002, confronté à des problèmes d'équilibre financier, le Conseil fédéral a approuvé un programme d'allègement budgétaire (PAB) de plusieurs milliards: celui-ci concerne également l'ODR, à raison de 140 millions jusqu'en 2006, dont 60 millions pour la première année.

Se refusant à opérer une coupe linéaire dans les forfaits alloués aux cantons pour la prise en charge des personnes du domaine de l'asile, déjà maintes fois revus à la baisse, l'ODR se propose d'exclure du régime de l'asile les personnes frappées d'une décision de non-entrée en matière; concrètement, cela comporte l'exclusion des prestations sociales des quelque 6000 personnes qui se trouvent dans cette situation. L'ODR fait valoir qu'après une décision de non-entrée en matière et à l'échéance du délai de départ, ces personnes séjournent illégalement dans le pays et violent de ce fait la Loi sur le séjour et l'établissement des étrangers (LSEE). Les autorités espèrent ainsi décourager les personnes concernées, qui finiront par quitter le pays sans engendrer de nouveaux coûts. Plus en général, elles misent sur l'effet dissuasif, pour les requérants potentiels, de l'attractivité moindre de la Suisse, puisqu'une demande manifestement infondée n'ouvrira pas de perspectives d'une aide sociale ou d'un séjour irrégulier dont on peut espérer une régularisation. D'autres pays, tels le Danemark et les Pays-Bas, ont déjà introduit des mesures analogues².

Les associations de défense du droit d'asile s'insurgent contre ces mesures, menant à la précarisation extrême de personnes exclues à la fois des prestations sociales et du marché du travail. Elles font valoir que dans la mesure où ces normes s'appliquent à des personnes particulièrement vulnérables (mineurs non accompagnés, femmes), elles pourraient être en opposition avec des conventions internationales, comme la Convention sur les droits de l'enfant ou la Convention européenne des droits de l'homme. Elles observent également que ces mesures comportent de graves risques d'augmentation des sans-papiers (Annuaire 2003, $\mathrm{n}^{\mathrm{o}} 1$, p. 219) et du travail au noir, ainsi que de recrudescence de la criminalité. Elles se demandent si, en fin de compte, les économies actuelles ne vont pas occasionner des coûts bien plus élevés, tant pour les collectivités locales que pour les œuvres d'entraide et les Eglises ${ }^{3}$.

2 Des observateurs relèvent que le succès de pareille disposition aux Pays-Bas a été de courte durée: si le nombre de requêtes a effectivement baissé initialement, au bout de deux ans, elles avaient atteint le même niveau qu'auparavant. «Du travail plutôt que l'aide sociale !», Planète Exil, nº 22, juin 2003.

3 Jürg Schertenleib, «Zu den Entlastungsmassnahmen im Asylbereich», Asyl, 3/03. 
Plus largement, divers milieux interprètent ces mesures comme une tentative de la Confédération de transférer sur les cantons les coûts de la prise en charge de ces personnes qui, une fois exclues du domaine de l'asile, ne manqueraient pas de tomber dans l'assistance. En effet, comme le message sur les mesures du PAB03 le reconnaît, ces personnes sont en droit de solliciter l'aide en situation de détresse que la Constitution fédérale garantit à l'article 12, une tâche qui incombe justement aux autorités locales. Cela explique le scepticisme des cantons, qui ont exprimé leur inquiétude lors de la Conférence des directeurs cantonaux des affaires sociales ${ }^{4}$.

Les autorités avaient souhaité une entrée en vigueur accélérée des mesures d'économie; le Conseil des Etats avait approuvé la proposition mais le Conseil national l'a refusée. Puisque l'élimination des divergences entre les deux Chambres ne pouvait avoir lieu avant la session d'hiver, le Conseil fédéral a renoncé à introduire ces mesures de manière urgente et recourt à la procédure ordinaire. Celle-ci semble d'ailleurs plus appropriée pour traiter d'une partie des mesures proposées (par exemple la limitation des délais de recours). Le sujet sera traité par les nouvelles Chambres fédérales élues le 19 octobre 2003; les mesures d'économie pourront entrer en vigueur au plus tôt en avril $2004^{5}$.

\section{Révision de la loi sur l'asile}

La révision de la loi sur l'asile a été discutée dans la Commission des institutions politiques du National en novembre 2003: celle-ci veut introduire une norme selon laquelle l'aide au développement pourrait être gelée partiellement ou entièrement pour ces Etats qui rechignent à récupérer leurs ressortissants renvoyés ${ }^{6}$. Par ailleurs, la commission n'a pas voulu lever l'interdiction de travail durant les trois premiers mois, malgré l'appel de la Ville de Zurich ${ }^{7}$. La loi sur l'asile sera fort probablement discutée en plénum durant la session spéciale de mai 2004, ensemble avec la nouvelle loi sur les étrangers.

\section{Une procédure accélérée: le système DUO}

La proposition de la suppression de l'aide sociale pour les requérants déboutés se greffe sur le renforcement des procédures accélérées à la frontière, connu sous le nom de DUO, qui intervient avant la répartition des requérants dans les cantons et qui vise à écarter les demandes n'ayant aucune chance d'aboutir.

A la mi-août, l'ODR tirait un bilan positif de la première année du système de procédure accélérée: les décisions de non-entrée en matière sont en augmentation. Un cinquième des décisions de première instance a été rendu directement dans les centres d'enregistrement et dans le centre de transit: la moitié des demandes ont été jugées infondées ${ }^{8}$. La nouvelle procédure est systématiquement appliquée aux requérants africains.

4 «Suppression de l'aide sociale aux requérants d'asile déboutés: les cantons expriment leur scepticisme», Le Temps, $1^{\text {er }}$ mars 2003.

5 DFJP, Programme d'allègement budgétaire 2003 : introduction des mesures prévues dans le domaine de l'asile reportée au $1^{\text {er }}$ avril 2004, communiqué de presse, 16 octobre 2003.

6 DFJP, Une commission du National durcit la loi sur l'asile. Aide au développement gelée pour les pays non coopératifs, communiqué de presse, 17 novembre 2003.

Voir plus bas, «Les propositions zurichoises en matière d'accueil des requérants d'asile».

ODR, Procédure d'asile accélérée. En un an, le système DUO fait ses preuves, communiqué de presse, 14 août 2003. 


\section{Publication de la liste des Etats tiers sûrs}

Le 25 juin 2003, le Conseil fédéral a publié la liste des pays sûrs, comme le réclamait l'UDC. Celle-ci comprend, à côté des pays de l'UE et de l'AELE, les dix pays qui vont rejoindre l'Union européenne en 2004, ainsi que la Bosnie et la Macédoine. La nouvelle liste comprend ainsi 38 pays alors que la précédente n'en comprenait que neuf: l'Albanie, la Bulgarie, la Gambie, le Ghana, l'Inde, la Lituanie, la Mongolie, la Roumanie et le Sénégal. La qualification d'Etat sûr fera l'objet d'un examen annuel. Ce principe permet d'accélérer et de simplifier la procédure d'asile. En effet, les autorités n'entrent pas en matière sur les demandes provenant de ces pays, sauf s'il existe des indices de persécution. Environ $10 \%$ des demandeurs d'asile proviennent de ces régions ${ }^{9}$.

L'OSAR se dit très inquiète de l'inclusion de la Bosnie et de la Macédoine dans la liste des Etats sûrs. Ce principe a été introduit en 1990: un Etat était considéré comme sûr lorsqu'il jouissait d'une grande stabilité, assurait un large respect des droits de l'homme et appliquait les conventions internationales en matière d'asile. Or, pareilles conditions ne semblent pas remplies dans ces deux pays balkaniques. L'OSAR demande que, comme cela est expressément prévu, le cas des personnes particulièrement vulnérables (malades, mineurs non accompagnés, membres de minorités) soit néanmoins examiné sans restrictions et que leur besoin de protection soit pris en compte ${ }^{10}$. L'examen par l'ODR des demandes de protection émanant des personnes particulièrement vulnérables de ces deux pays est d'autant plus crucial que, dans le cadre des mesures d'allègement budgétaire, une non-entrée en matière entraîne l'exclusion du système d'assistance, qui jetterait à la rue des personnes nécessitant protection.

L'inclusion de la Bosnie et de la Macédoine parmi les Etats sûrs ne modifie cependant en rien la situation de leurs ressortissants déjà au bénéfice d'une admission provisoire ou du statut de réfugié; par contre, ceux qui ont obtenu une réponse négative à leur requête doivent rentrer dans leur pays.

\section{$\square$ Mesures de politique sociale}

\section{Les propositions zurichoises en matière d'accueil des requérants d'asile}

La défaite évitée de justesse lors de la votation sur l'initiative UDC en novembre 2002 a été une sonnette d'alarme, indiquant l'aggravation de la perception des requérants d'asile en Suisse. Les autorités de la Ville de Zurich, confrontées au quotidien avec l'exécution des mesures fédérales et la prise en charge des requérants d'asile, ont voulu réagir à cette dégradation avec des propositions positives: s'opposant à la tendance dominante d'une réglementation toujours plus répressive et restrictive en matière d'asile, elles ont dressé un décalogue de mesures pour sortir de l'impasse.

Elles proposent en particulier d'alléger la charge qui pèse sur l'assistance aux requérants d'asile en favorisant l'accès à l'emploi des requérants d'asile qui sont interdits de travail pendant une période variable entre trois et six mois selon les cantons. Lorsqu'ils travaillent, ils sont obligés de prendre les emplois pour les-

ODR, Asile: désignation de nouveaux «pays sûrs », communiqué de presse, 25 juin 2003.

10 OSAR, L'OSAR demande protection des personnes vulnérables, communiqué de presse, 31 juillet 2003. 
quels aucun autre Suisse ou étranger au statut plus favorable n'a postulé; ils sont confinés dans certains secteurs, notamment l'hôtellerie, où les salaires sont notoirement bas. Il n'est dès lors pas étonnant que des working poor, grevés d'un impôt particulier, les comptes de sûreté ${ }^{11}$, dépendent de l'assistance. Une occupation aurait des effets bénéfiques pour les requérants d'asile, qui seraient ainsi incités à se prendre en charge, et améliorerait leur image - on leur reproche de «se faire payer à ne rien faire». Les autorités locales peuvent promouvoir des emplois d'utilité publique, pour soutenir l'activité professionnelle des requérants d'asile. Le système des comptes de sûreté, qui s'est révélé très coûteux, serait à remplacer afin de faire diminuer le nombre de requérants d'asile qui, en raison de leur revenu écourté par ces paiements, dépendent de l'assistance. Les enfants et les jeunes devraient avoir accès à la scolarité obligatoire et à la formation, conformément au droit garanti par la Constitution (article 62). La Confédération devrait soutenir financièrement les autorités locales engagées dans une politique de ce type. Enfin, le Conseil communal de Zurich en appelle à la convocation d'une conférence nationale sur l'asile ${ }^{12}$.

Le Manifeste de Zurich a reçu un accueil plutôt positif. La Ville a commencé à traduire en pratique ses propres propositions et la Confédération se dit disposée à assumer une partie des coûts occasionnés. L'expérience passée des programmes d'occupation a été concluante: ils ont joué en faveur d'une meilleure intégration des requérants d'asile, une moindre criminalité et une amélioration de l'image des requérants d'asile auprès de la population. Cependant, l'ODR juge inopportun de créer des places de travail par voie administrative et préfère que les requérants trouvent des opportunités d'emploi dans l'économie afin, entre autres, d'éviter une attractivité accrue de la Suisse aux yeux des requérants ${ }^{13}$.

La Conférence nationale sur l'asile au début avril 2003 ne s'est pas concentrée sur les propositions zurichoises, mais a offert à la responsable du Département fédéral de justice et police, Ruth Metzler, la possibilité d'illustrer son projet de concrétisation des mesures d'allègement budgétaire.

\section{Action humanitaire: un bilan nuancé}

L'ODR tire un bilan de son dispositif visant à octroyer un permis humanitaire aux «anciens » requérants d'asile, entrés en Suisse avant la fin 1992 (HUMAK, «Action humanitaire 2000»; Annuaire 2001, pp. 281-282). Quelque 16’000 personnes ont reçu un permis $\mathrm{F}$ (admission provisoire); le permis ainsi obtenu constitue une phase transitoire en attendant d'obtenir un permis B qui représente la première véritable consolidation de leur statut. Par contre, il n'a pas été possible de faire rentrer chez eux tous ceux dont la requête liée à la HUMAK avait été rejetée (700). Dans la mesure où il n'a pas été possible de régler tous les cas en suspens, il pourra s'avérer nécessaire à moyen terme de lancer une nouvelle action ${ }^{14}$.

11 «Du travail que les Suisses ne veulent pas faire», Bilan, $1^{\text {er }}$ mai 2003. Selon Marcello Fontana, de l'ODR, «le salaire moyen d'un requérant d'asile actif en Suisse s'élève à 2500 francs par mois. La Confédération retire $10 \%$ de son salaire brut».

12 Aufruf des Stadtrates von Zürich: Zehn Regeln für eine neue Schweizerische Asylpolitik, 31. Januar 2003, <www.stzh.ch/str/aufruf_03/pdf/StR_ZH_Asylpolitischer_Aufruf.pdf>.

13 Jörg Frieden, Der Asylbereich. Entwicklung und sozialpolitische Perspektiven aus der Sicht des Bundes, Rede an der Konferenz der Städteinitiative, Freiburg, 9. Mai 2003.

14 «Action humanitaire 2000 : un bilan en demi-teinte», Asylon, octobre 2003. 
La consolidation du statut des personnes présentes dans le pays depuis de nombreuses années est également au centre des préoccupations de la Commission fédérale contre le racisme (CFR). Dans un rapport rendu public à la fin octobre $2003^{15}$, la CFR critique le permis F tel qu'il existe actuellement. Ce permis est octroyé aux requérants d'asile qui, bien que ne remplissant pas les conditions pour la reconnaissance du statut de réfugié aux termes de la Convention de Genève, sont reconnus comme ayant un droit légitime à une forme de protection subsidiaire, ainsi qu'aux personnes relevant de l'asile qui ne peuvent être renvoyées et/ou ont établi des liens solides avec la Suisse. Or, l'étude révèle que $60 \%$ d'entre eux résident en Suisse depuis plus de cinq ans et que $30 \%$ des adultes sont là depuis plus de dix ans. En dépit de l'interdiction du regroupement familial, $45 \%$ d'entre eux sont des enfants. Absence de mobilité géographique et professionnelle, confinement dans certains secteurs d'activité professionnelle, soumission à la priorité à la main-d'œuvre indigène dans leur recherche d'une place d'apprentissage pour les jeunes, aide sociale selon des barèmes réduits: autant de dispositions qui rendent précaire l'insertion d'une frange importante de 26'000 porteurs d'un permis F destinés pourtant à rester dans le pays à plus longue échéance. La proposition, contenue dans le projet de révision partielle de la loi sur l'asile, d'instituer une «admission à titre humanitaire» (Annuaire 2003, $\mathrm{n}^{\mathrm{o}}$ 1, pp. 212-213) montre que l'analyse des insuffisances de l'actuelle «admission provisoire» est partagée par les autorités.

\section{$\square$ Politique extérieure en matière de migration}

Au chapitre de la politique extérieure en matière d'asile, il faut relever la poursuite de la politique de signature d'accords de réadmission avec les pays de provenance des réfugiés ${ }^{16}$. A cet effet, la Suisse a signé un accord de ce type avec le Nigeria en janvier 2003, inspiré de ceux signés avec des Etats européens (Annuaire 2000, p. 265). Celui-ci prévoit une coopération dans le domaine migratoire, avec notamment la formation d'employés des autorités nigérianes d'immigration et des programmes d'aide au retour de ressortissants nigérians ${ }^{17}$; il est également question d'une collaboration en matière de lutte contre le sida ${ }^{18}$.

Par contre, au mois de mars 2003, le Sénégal a refusé de ratifier l'accord de transit signé le 8 janvier à Dakar. Contrairement aux accords de réadmission, qui concernent les ressortissants du pays signataire du texte, l'accord de transit concernait les requérants africains déboutés provenant de l'Afrique de l'Ouest. Cet accord se voulait une réponse à la difficulté d'identifier les ressortissants africains qui se présentent comme requérants d'asile. Il prévoyait que les requérants d'asile africains dont on n'avait pu établir l'identité séjourneraient trois jours dans la zone de transit de l'aéroport de Dakar pour faciliter sur place

15 Martina Kamm, Denise Efionayi-Mäder, Anna Neubauer, Philippe Wanner, Fabienne Zannol, Aufgenommen aber ausgeschlossen? Die vorläufige Aufnahme in der Schweiz, Neuchâtel, Forum suisse pour l'étude des migrations et de la population, 2003.

16 Entre 1993 et 2003, la Suisse a signé des accords de réadmission avec 20 pays: Albanie, Allemagne, Autriche, Bosnie-Herzégovine, Bulgarie, Croatie, Estonie, France, Hongkong, Hongrie, Italie, Lettonie, Liechtenstein, Lituanie, Macédoine, Nigeria, Roumanie, Sénégal, Sri Lanka et Yougoslavie.

17 DFJP, Le Conseil fédéral approuve un accord avec le Nigeria, communiqué de presse, 10 septembre 2003.

18 «Asyl-Experiment ohne Gewähr. Offene Fragen zu den Verträgen mit Senegal und Nigeria», Neue Zürcher Zeitung, 11. Januar 2003. 
l'identification de leur nationalité et qu'ils seraient reconduits en Suisse en cas d'échec de ces vérifications. Les observateurs font remarquer que ce type d'accord est inédit au niveau international, ce qui rend d'autant plus difficile une appréciation des chances et des risques de cet instrument ${ }^{19}$.

Au Sénégal, la pression des ONG contre un accord négocié au simple niveau ministériel, qui risquait de faire du pays la «poubelle de l'Afrique de l'Ouest $»^{20}$ et de lui aliéner les sympathies des pays limitrophes face à d'éventuels «charters de l'immigration", a eu raison de cette tentative de développement d'une politique extérieure de migration en Afrique ${ }^{21}$. De son côté, la République démocratique du Congo a refusé, en septembre 2003, l'autorisation d'atterrir à un vol affrété pour un rapatriement forcé ${ }^{22}$. De tels efforts pour une politique extérieure de migration restent néanmoins à l'ordre du jour, comme l'atteste l'issue de l'accord avec le Nigeria.

Toujours au chapitre des relations internationales, mais cette fois-ci au niveau européen, relevons qu'en janvier 2003 est entré en fonction le système Eurodac, la banque de données sur les empreintes digitales qui constitue l'instrument technique principal du système de surveillance européen en matière de demandeurs d'asile. Cela rend d'autant plus intéressant pour la Suisse l'avancement du dossier Dublin (Annuaire 2003, $\mathrm{n}^{\circ}$ 1, p. 211), qui fait partie des matières traitées dans le cadre des bilatérales II entre la Suisse et l'Union européenne.

Enfin, la Suisse a décidé de ne pas signer la Convention internationale sur la protection des droits de tous les travailleurs migrants et des membres de leur famille (MWC), qui affirme la prohibition des discriminations raciales et de nationalité à l'encontre des migrants. Cette convention, entrée en vigueur le

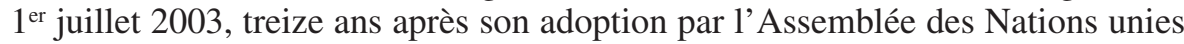
en 1990, n’a pas encore été signée par un Etat de l'OCDE.

\section{$\square$ La politique d'information de l'ODR}

L'ODR a multiplié ses efforts de communication sur un thème qui continue d'être au cœur des débats publics (Annиaire 2000, p. 266). Après un lifting radical de ses publications, il s'est engagé dans les contacts avec le public lors de manifestations commerciales et de comptoirs ${ }^{23}$. Il a également développé un jeu virtuel sur l'asile, swiss-checkin.ch, destiné en priorité aux jeunes et visant à transmettre de manière ludique l'information sur l'asile.

Alors que l'ODR était plutôt satisfait de l'impact de ce jeu, la Commission fédérale contre le racisme et les milieux de défense du droit d'asile l'ont critiqué: bien que n'étant pas raciste, ce jeu utilise de manière subtile la distance sociale et émotionnelle des Suisses vis-à-vis des réfugiés et la renforce. Par là, le jeu virtuel incite au rejet et à l'exclusion des requérants d'asile ${ }^{24}$. En raison de ces critiques, l'ODR a assez rapidement préféré retirer le jeu de son site.

19 Ibid.

20 «Abdoulaye Wade: l'Afrique n'est pas un continent où il suffit d'injecter de temps et temps des fonds », Le Temps, 3 juin 2003.

21 Les premiers pas vers une politique extérieure de migration étaient liés avant tout à la prévention des migrations (Annuaire 2000, p. 265); ici, il s'agit de gestion des flux existants.

22 «Kinshasa refuse un avion rempli de réfugiés congolais déboutés », Le Courrier, 29 septembre 2003.

23 «L'ODR se livre au grand public », Asylon, mars 2003.

24 «L'asile n'est pas un jeu», Vivre ensemble, nº 93, juin 2003. 
La loi sur les étrangers (Annuaire 2001, pp. 288-289) n'aboutira pas de sitôt en discussion parlementaire. La procédure de consultation relative à cette loi avait montré une forte polarisation des positions (Annuaire 2002, p. 235), de sorte que l'on s'attend à un renvoi des délibérations en la matière. Les milieux patronaux avaient souhaité connaître l'impact de la libre circulation des personnes avec les pays de l'UE avant de se prononcer. Aujourd'hui, l'élargissement de l'Union européenne en mars 2004 met sur le devant de la scène la question de l'extension de la libre circulation aux ressortissants de ces pays.

\section{$\square$ Bilan de la libre circulation avec l'UE}

En dépit des appréhensions qu'ils avaient suscitées, les accords bilatéraux entre la Suisse et l'UE entrés en vigueur le $1^{\text {er }}$ juin 2002 n'ont pas provoqué de changements majeurs dans les flux migratoires. Pas d'invasion des ressortissants de l'UE, ni de déstabilisation du marché de l'emploi ou de dumping salarial ${ }^{25}$ : quelque $15^{\prime} 000$ Européens sont venus travailler ou ont régularisé leur situation. La phase est certes encore transitoire, avec un régime d'autorisations de travail obligatoires, de priorité pour la main-d'œuvre indigène et de contrôle des salaires. Mais, dès juin 2004, les mesures d'accompagnement contre la sousenchère salariale et sociale prendront le relais. L'accord qui permet la libre circulation est aujourd'hui soumis à révision pour décider des modalités d'intégration des dix pays qui rejoignent l'UE en 2004.

\section{$\square$ L'élargissement de l'UE à l'Est}

Il semble que passé les premières craintes quant à la libre circulation, les appréhensions se soient reportées sur la nouvelle étape, celle relative à l'extension de la libre circulation aux ressortissants des dix nouveaux pays de l'UE. Les autorités saluent cet élargissement comme une contribution à la pacification du continent européen et voient l'extension du marché européen à 450 millions de personnes comme une promesse de croissance économique. De plus, les nouveaux membres de l'UE offrent un niveau de formation élevé et disposent d'une maind'œuvre qualifiée, mais aussi de travailleurs susceptibles de couvrir les besoins de l'agriculture et du tourisme en Suisse.

Le 14 mai 2003, le Conseil fédéral a mis en consultation le mandat de négociation concernant cette extension, négociation qui vise à obtenir des dispositions transitoires appropriées, analogues à celles prévues par les quinze pays membres de l'UE actuelle qui ont adopté des dispositions transitoires pendant les premiers sept ans en matière de libre circulation de la main-d'œuvre. Le dossier n'est pas des plus faciles, dans la mesure où la main-d'œuvre de l'Est est recherchée par certains milieux et crainte par d'autres.

L'USS se dit fondamentalement favorable à l'élargissement, mais le Syndicat de l'industrie et du bâtiment craint la sous-enchère salariale et sociale, et revendique de nouvelles mesures de sauvegarde. Il demande des périodes de transition aussi longues qu'en Allemagne et en Autriche, la conclusion de conventions

25 Bureau de l'intégration Suisse-UE, Accords bilatéraux I: premières expériences un an après leur entrée en vigueur, communiqué de presse, 27 mai 2003. 
collectives dans des secteurs qui en sont dépourvus (agriculture, nettoyage, transports) et l'adoption en Suisse de certaines directives européennes en matière de droit du travail ${ }^{26}$. De l'autre côté de l'échiquier politique, l'UDC s'oppose à l'extension des accords de libre circulation et annonce d'ores et déjà un référendum ${ }^{27}$.

Ainsi, la ratification de cet accord, prévue au plus tôt pour début 2005, fait déjà débat. L'enjeu est de taille car il suffit d'un vote négatif sur ce dossier pour que les sept accords bilatéraux de 1999 soient dénoncés.

\section{$\square$ Les sans-papiers: un dossier en suspens}

La question des sans-papiers hante le débat sur la politique d'admission. Si la circulaire Metzler de décembre 2001 avait laissé espérer une voie de sortie (Annuaire 2003, n 1, pp. 220-221), aujourd'hui on déchante. Ainsi, seules 500 personnes ont été régularisées depuis septembre 200128, sur les milliers qui se trouvent sans statut légal (entre 70'000 et 180'000 selon une estimation). Cet état de fait est insatisfaisant aux yeux de certains acteurs politiques. Ainsi, le Grand Conseil genevois a approuvé une motion, présentée par l'ensemble de l'échiquier politique, demandant la régularisation; il se joint ainsi à une démarche du Conseil d'Etat allant dans le même sens. Celui-ci, en effet, avait écrit en octobre 2003 un courrier adressé au Collectif de soutien aux sans-papiers, dans lequel il dénonce les faiblesses de la politique migratoire suisse en matière d'admissions et saisit le Conseil de surveillance du marché de l'emploi pour qu'il se prononce sur cette question, qui relève de la gestion du marché du travail.

Caritas, quant à elle, taxe de peu efficace et insuffisante l'actuelle procédure de régularisation «des cas de rigueur» et se prononce résolument pour une régularisation collective des sans-papiers qui ne relèvent pas du domaine de l'asile ${ }^{29}$. Elle demande en même temps une certaine ouverture du marché suisse de l'emploi aux travailleurs des Etats non membres de l'UE et de l'AELE.

\subsection{Politique d'intégration}

En trois ans on prend racine: tel pourrait être le slogan de la politique d'intégration, qui fait désormais partie intégrante du paysage de la politique suisse. Cette année, deux débats dominent l'actualité: la nouvelle orientation de la politique active d'intégration et l'aggiornamento du concept et des pratiques de naturalisation. Par ailleurs, l'impasse dans laquelle se trouve le dossier des sans papiers suscite des réactions dans divers milieux.

26 Jean-Claude Rennwald, «Immigration, le vrai débat», L'Evénement syndical, mai 2003.

27 La Commission des institutions politiques du Conseil national a approuvé en mai 2003 le principe selon lequel les agriculteurs et les hôteliers suisses peuvent recourir à des travailleurs des pays de l'Est non qualifiés. Il est question d'un statut transitoire valable jusqu'à l'adoption formelle de l'élargissement de l'UE, prévoyant un séjour maximal de six mois par an pour des travailleurs qui ne bénéficient pas du regroupement familial. Certains conseillers nationaux UDC ne voient pas d'un mauvais œil cette proposition. «L'idée d'un statut pour les travailleurs de l'Est met en lumière la position ambiguë de l'UDC», Le Temps, 20 mai 2003.

$2890 \%$ des demandes de régularisation déposées proviennent des cantons de Genève, Vaud, Neuchâtel, Fribourg et Berne.

29 «Neuer Ruf nach Bewilligungen für Papierlose. Caritas Schweiz für bedingte Regularisierung», Neue Zürcher Zeitung, 12. Dezember 2003. 


\section{$\square$ Ordonnance d'intégration}

Suite à l'approbation de l'article sur l'intégration des immigrés dans la Loi sur le séjour et l'établissement des étrangers (LSEE) (Annuaire 2001, p. 290), la Confédération s'est vu attribuer la compétence de favoriser l'intégration: pour les années 2002 et 2003, la Commission fédérale des étrangers (CFE) dispose d'un crédit de 12,5 millions. Il est maintenant question d'une révision partielle de l'ordonnance d'application d'octobre 2000.

Les autorités souhaitent en effet introduire rapidement des nouveautés, sans attendre que la nouvelle loi sur les étrangers parvienne à accomplir son long et accidenté chemin parlementaire. Le texte, actuellement en procédure de consultation, définit les nouvelles priorités pour les projets financés par la Confédération: promouvoir la compréhension (à travers des cours de langue), ouvrir les institutions à la population étrangère, faciliter la cohabitation, développer des centres de compétence et favoriser l'innovation et le contrôle de qualité des projets soutenus ${ }^{30}$.

L'ordonnance comporte en outre deux innovations majeures. En premier lieu, elle étend le champ d'application à des groupes immigrés n'étant pas détenteurs d'un permis durable (permis B ou C) $)^{31}$. Il s'agit de rendre accessible aussi aux titulaires d'un permis $\mathrm{F}$ le dispositif des mesures d'intégration qui se met en place depuis 2000. Cette proposition est cohérente avec la modification proposée dans la révision partielle de la loi sur l'asile, qui prévoit l'institution d'une admission à titre humanitaire pour les personnes relevant de l'asile dont le renvoi est non exigible ou illicite et qui, en conséquence, sont appelées à rester en Suisse à moyen ou à long terme. Ainsi, les seuls requérants d'asile qui seront exclus des mesures d'intégration sont ceux pour lesquels aucune décision définitive n'a été rendue ou qui ont obtenu l'admission à titre provisoire suite à l'impossibilité d'exécuter le retour.

En deuxième lieu, à travers l'ordonnance, les autorités souhaitent ancrer le concept de la réciprocité du processus d'intégration entre immigrés et société de résidence, en sollicitant davantage de contribution de la part des immigrés. Il est ainsi exigé de ces derniers qu'ils respectent l'ordre juridique suisse et fassent des efforts en vue d'acquérir des connaissances de la langue locale. Un système de récompenses et de sanctions permet d'honorer ou, au contraire, de sanctionner le comportement des immigrés, notamment avec l'octroi anticipé d'une autorisation d'établissement. L'exigence de connaître la langue locale devient une obligation pour les personnes qui envisagent d'exercer en Suisse une fonction publique, tels l'assistance dans le domaine religieux ou l'enseignement des langue et culture du pays d'origine ${ }^{32}$.

30 IMES (Office fédéral de l'immigration, de l'intégration et de l'émigration - nouveau nom, dès le $1^{\mathrm{er}}$ mai 2003, de l'Office fédéral des étrangers OFE), Promotion de l'intégration des étrangers. Nouvel ordre de priorité, communiqué de presse, 23 mai 2003.

31 Ce faisant, la nouvelle ordonnance prend en compte les critiques soulevées par les milieux de l'immigration organisée quant à la limitation du champ d'application (Annuaire 2001, p. 291) et tient dûment compte de l'évolution de la population relevant de l'asile, notamment des permis F (voir plus haut, «Action humanitaire: un bilan nuancé»).

32 Procédure de consultation. Révision partielle de l'ordonnance sur l'intégration des étrangers (OIE; RS 142.205), rapport explicatif, mai 2003. 
Enfin, pour favoriser la coordination et les échanges horizontaux d'expériences au niveau communal en vue de l'intégration, l'OFE a institué en février 2003 une Conférence suisse des délégués à l'intégration des communes, des régions et des cantons. La Conférence tripartite sur les agglomérations, quant à elle, assure une coordination verticale entre Confédération, cantons et communes: elle a recommandé l'institution d'organismes interdépartementaux à l'échelon infranational.

Par ailleurs, toujours en anticipant sur la nouvelle loi sur les étrangers (LEtr), la nouvelle Loi sur la formation professionnelle (nLFPr), qui entrera en vigueur le $1^{\text {er }}$ janvier 2004, provoque une modification de l'actuelle loi sur les étrangers (LSEE) en vue de favoriser le regroupement familial précoce des enfants et garantir l'accès à la formation professionnelle des adolescents venus en Suisse dans le cadre du regroupement familial.

\section{$\square$ Naturalisations : le Conseil des Etats et le Tribunal fédéral se prononcent}

En juin 2003, en suivant le Conseil national, le Conseil des Etats s'est prononcé, à une confortable majorité, en faveur de la naturalisation facilitée pour les jeunes de la deuxième génération et d'une naturalisation en fonction du droit du sol pour la troisième génération. Si tous étaient d'accord pour que la naturalisation jure soli prenne effet à la naissance, une minorité aurait souhaité qu'elle intervienne sur demande des parents. Finalement, le texte approuvé accorde la nationalité suisse automatiquement à la naissance, à moins que les parents ne s'y opposent; cette procédure rend ainsi possible le maintien de la nationalité d'origine dans de nombreux cas de figure (Annuaire 2003, $\mathrm{n}^{\circ}$ 1, p. 222). Dans la mesure où ces dispositions nécessitent une modification de la Constitution, cette matière sera soumise au scrutin populaire.

Le Conseil des Etats a refusé par contre l'introduction du droit de recours pour les candidats déboutés en matière de naturalisation, une proposition qui s'inspire de la Convention européenne sur la naturalisation. Les débats ont été très vifs sur ce point, d'aucuns soulignant que le recours au judiciaire contre une décision prise par vote populaire mettait en danger l'édifice de la démocratie directe. Entre-temps, suite à la sentence du Tribunal fédéral (voir plus bas), le Conseil fédéral a retiré de la loi sur la naturalisation ce dispositif de naturalisation par le peuple ${ }^{33}$.

Le 29 juin 2003, les électeurs d'Emmen ont encore une fois refusé la naturalisation à des candidats provenant des Balkans, en acceptant par contre les Italiens et autres groupes de ressortissants extracommunautaires ${ }^{34}$; quelques jours plus tard, le Tribunal fédéral (TF) devait se prononcer en séance publique sur le recours de cinq candidats déboutés en mars 2000 en votation populaire à Emmen (Annuaire 2001, pp. 291-292).

En premier lieu, les juges du TF se sont prononcés contre l'initiative de l'UDC qui visait à soumettre les naturalisations à l'approbation des citoyens en ville de Zurich; leur décision confirme l'avis du Conseil d'Etat zurichois, qui avait conclu à l'inconstitutionnalité de cette initiative. En deuxième lieu, de manière

33 «Akt der Realpolitik. Kein gesetzliches Rekursrecht bei Nichteinbürgerung », Neue Zürcher Zeitung, 22. August 2003.

34 «Emmen refuse de naturaliser des citoyens d'ex-Yougoslavie», Le Temps, $1^{\text {er }}$ juillet 2003. 
cohérente avec la première décision, le Tribunal fédéral a accepté le recours des recalés d'Emmen. Il a de la sorte estimé que la procédure de naturalisation doit obéir aux règles fondamentales qui encadrent toute décision administrative, et que ces règles sont incompatibles avec la naturalisation par le peuple ${ }^{35}$. Il a ainsi décrété qu'il est inapproprié de se servir d'un instrument de la démocratie directe lorsqu'il s'agit d'appliquer des normes à des cas individuels. La première Cour de droit public du Tribunal fédéral a décidé à l'unanimité que la procédure consistant à soumettre les naturalisations au scrutin populaire viole le droit constitutionnellement garanti des candidats à obtenir une décision motivée. Le Tribunal fédéral a cependant évité de statuer sur les décisions prises en assemblée communale, qui ne permettent pas non plus une motivation du refus de naturalisation.

Dans ses considérants adoptés à l'unanimité, le Tribunal fédéral fait valoir que les autorités trouvent une limite à leur pouvoir d'appréciation dans l'interdiction de la discrimination prononcée par la Constitution à l'article 8. Les autorités doivent protéger les droits fondamentaux, dont le droit d'être entendu et celui du respect de la vie privée. En outre, la démocratie directe est applicable dans le cas de décisions qui concernent potentiellement l'ensemble des citoyens, mais elle ne permet pas une garantie adéquate des droits fondamentaux des individus; la démocratie directe trouve ainsi une limite dans les droits garantis constitutionnellement ${ }^{36}$. Le TF invite les communes à définir une procédure de naturalisation respectueuse des droits fondamentaux.

Saluée par les milieux proches de l'immigration aussi bien que par la Commission fédérale des étrangers et la Commission fédérale contre le racisme, la décision a provoqué une levée de boucliers dans les dizaines de communes alémaniques qui doivent désormais changer leur procédure de naturalisation.

La décision des juges fédéraux est toutefois controversée: des politiciens d'envergure ont regretté que «le juridique prenne le pas sur le politique» ${ }^{37}$. Des observateurs font valoir que «la décision de principe tout à fait convaincante sur le plan technique a frappé le nerf de la démocratie directe chez un nombre non négligeable de citoyens et ceci ne laisse pas voir la fin de la tension entre Etat de droit et démocratie $»^{38}$. Puisque le Tribunal fédéral ne peut qu'examiner la conformité des actes juridiques avec les lois existantes, c'est le peuple et le législateur qui conditionnent en dernière instance le pouvoir du judiciaire. Le TF met cependant son espoir dans les vertus pédagogiques de son jugement pour que le législateur se conforme aux principes de l'Etat de droit.

En réaction à la sentence du Tribunal fédéral, qualifiée de «scandaleuse», l'UDC se propose de lancer une initiative au mois de mars 2004 sur le thème des naturalisations, afin que les communes puissent définir de manière autonome quel organe est responsable des décisions de naturalisation ${ }^{39}$.

35 «Zwei Machtworte zur Einbürgerung. Diskriminierung verboten - Begründung erforderlich», Neue Zürcher Zeitung, 10. Juli 2003.

36 «Kein rechtsfreier Raum bei Einbürgerungen. Bundesgericht begründet Urteile», Neue Zürcher Zeitung, 25. Juli 2003

37 Ainsi Arnold Koller, à l'origine de l'actuelle Constitution, dans une interview à la Sonntagszeitung. «Les considérants du TF ne clarifient qu'une partie des problèmes », Le Courrier, 25 juillet 2003.

38 «Das Bundesgericht zwischen Recht und Macht», Neue Zürcher Zeitung, 5. August 2003.

39 «Les délégués du parti lancent deux initiatives sur l'asile et les naturalisations », Le Temps, 15 septembre 2003. 


\section{$\square$ Droits politiques: des pas hésitants}

Le dossier des droits politiques aux étrangers avance avec de nombreuses hésitations. Le Grand Conseil vaudois a adopté les modifications de la Loi sur l'exercice des droits politiques, qui entre en vigueur le $1^{\text {er }}$ janvier 2004, afin de permettre l'application du droit de vote communal aux étrangers introduit par la nouvelle Constitution. En même temps, cependant, un comité se proclamant indépendant a lancé un référendum contre ce droit, pourtant accepté en votation populaire, lors de l'approbation de la nouvelle Constitution ${ }^{40}$. Par ailleurs, la droite bernoise s'oppose à la proposition du gouvernement de donner la possibilité aux communes d'accorder le droit de vote, voire celui d'éligibilité, aux étrangers. Par contre, à Genève, une initiative en faveur du droit de vote, voire d'éligibilité, sur le plan communal a abouti ; l'objet sera soumis en votation probablement l'année prochaine.

Selon l'avis de nombreux observateurs, l'UDC doit sa montée en puissance à sa manière de thématiser la présence de la population étrangère. Or, ce parti est devenu, avec les élections du 19 octobre 2003, le premier parti du pays en nombre de sièges au Conseil national: ceci ne manquera pas d'induire une modification des accents politiques sur les questions inhérentes à la présence d'étrangers en Suisse. L'UDC entend maintenir la pression sur ce sujet: elle avait annoncé avant les élections qu'elle voulait lancer une initiative sur le droit d'asile, la troisième du genre. Le projet demande le renvoi de toute personne ayant transité par un Etat tiers, sans tenir compte des objections du pays en question, la non-entrée en matière sur la demande de personnes arrivées en Suisse sans papier d'identité ou clandestinement, et l'expulsion des requérants délinquants. Par ailleurs, outre les requérants d'asile, l'UDC affûte ses armes contre une nouvelle cible: elle vise en effet à faire du séjour clandestin en Suisse un délit pénal puni de l'emprisonnement et de l'expulsion immédiate ${ }^{41}$. Enfin, l'arrivée au Conseil fédéral de Christoph Blocher, la figure de proue de l'UDC, à la tête, depuis $1^{\text {er }}$ janvier 2004, du Département fédéral de justice et police, responsable des questions d'asile et de migration, ainsi que le départ de l'actuel directeur de l'Office fédéral des réfugiés, Jean-Daniel Gerber, annoncent un tournant dans cette délicate matière.

40 «Une initiative populaire veut effacer le vote des étrangers de la Constitution», Le Courrier, 18 juin 2003.

41 «L'UDC veut replacer l'asile dans la campagne», Le Temps, 11 juillet 2003. 


\section{SOURCES}

Office fédéral des réfugiés, Asylon, parutions en 2002-2003.

Office fédéral des réfugiés, communiqués de presse, parutions en 2002-2003.

Office fédéral des réfugiés, Statistique en matière d'asile 2002, janvier 2003.

Augenauf, Bulletin, Berne, parutions en 2002-2003.

Centres de contact Suisses-immigrés et SOS Racisme, Carrefour, parutions en 2002-2003.

Organisation suisse d'aide aux réfugiés, Planète Exil, parutions en 2002-2003.

Schweizerische Flüchtlingshilfe, Asyl, Schweizerische Zeitschrift für Asylrecht und Praxis, Berne, parutions en 2002-2003.

Schweizerische Flüchtlingshilfe, Chronologie der Flüchtlingspolitik, parutions en 2002-2003.

Vivre ensemble (Centre de documentation sur le droit d'asile), Vivre ensemble, bulletin de liaison pour la défense du droit d'asile, Genève, parutions en 2002-2003.

\section{SITES INTERNET}

Haut-Commissariat des Nations unies pour les réfugiés : <www.unhcr.ch>.

Administration fédérale: <www. admin.ch>.

Office fédéral de l'immigration, de l'intégration et de l'émigration (ancien Office fédéral des étrangers): $<$ www.bfa.admin,ch>.

Office fédéral des réfugiés : <www. asyl.admin.ch $>$; <www. odr.admin.ch $>$.

Institut de science politique de l'Université de Berne: <www.anneepolitique.ch>.

Association romande contre le racisme: <www.AcorSosRacisme.org $>$.

European Council on Refugees and Exiles (ECRE), coordination européenne des organisations non gouvernementales dans le domaine des requérants d'asile et des réfugiés : <www.ecre.org $>$.

Human Rights Watch: <www.hrw.org >.

Menschenrechte Schweiz MERS - Praktische Hilfsinstrumente für die Bildungsarbeit zu Menschenrechtsthemen: <www.humanrights.ch>.

Organisation suisse d'aide aux réfugiés : <www.sfh-osar.ch $>$.

Solidarité sans frontières (coopération de BODS et Asylkoordination) : <www.sosf.ch $>$.

Vivre ensemble (Centre de documentation sur le droit d'asile) : <www.asile.ch $>$.

Le Courrier : <www.lecourrier.ch>. 